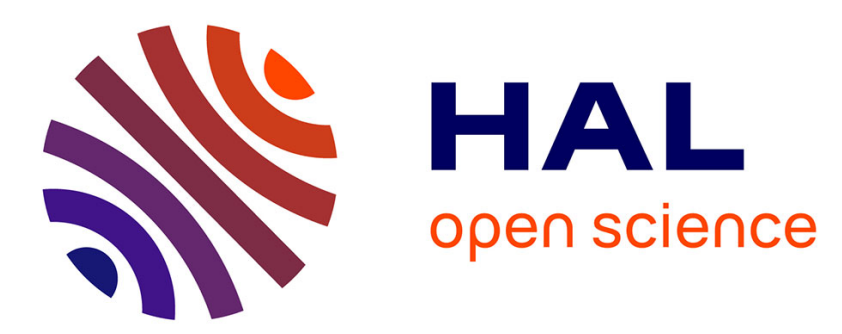

\title{
Karsts et paléokarsts du bassin de Mialet (Bordure Cévenole, Gard) : formation et évolution d'un karst démantelé
}

\author{
Laurent Bruxelles
}

\section{To cite this version:}

Laurent Bruxelles. Karsts et paléokarsts du bassin de Mialet (Bordure Cévenole, Gard) : formation et évolution d'un karst démantelé. KARSTOLOGIA, 1997, 30 (1), pp.15-24. hal-02017920

\section{HAL Id: hal-02017920 \\ https://hal-univ-tlse2.archives-ouvertes.fr/hal-02017920}

Submitted on 28 Feb 2019

HAL is a multi-disciplinary open access archive for the deposit and dissemination of scientific research documents, whether they are published or not. The documents may come from teaching and research institutions in France or abroad, or from public or private research centers.
L'archive ouverte pluridisciplinaire HAL, est destinée au dépôt et à la diffusion de documents scientifiques de niveau recherche, publiés ou non, émanant des établissements d'enseignement et de recherche français ou étrangers, des laboratoires publics ou privés. 


\title{
Karsts et paléokarsts du bassin de Mialet (Bordure Cévenole, Gard) :
}

\author{
Formation et évolution d'un karst démantelé
}

\author{
Laurent BRUXEUES
}

GDR 1058 et URA 903 du CNRS, Université de Provence

29 av. Robert Schuman - 13100 Aix-en-Provence cedex 1 / e-mail : Bruxelles1@aol.com

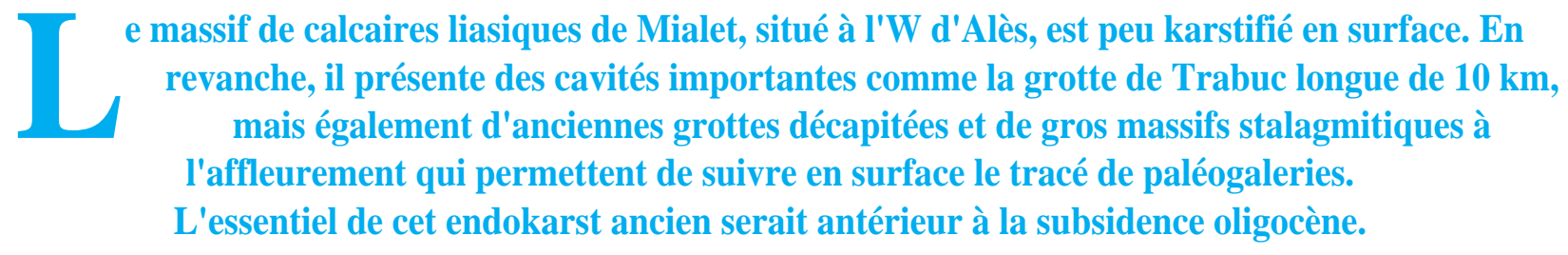

RÉSUMÉ : Le bassin de Mialet est un massif calcaire de $62 \mathrm{~km}^{2}$ appartenant à la bordure cévenole. Les phénomènes karstiques de surface sont rares, en revanche l'endokarst est bien développé et de nombreuses cavités de section importante existent à toutes altitudes qui débouchent à flanc de versant. La présence de poches de sédiments endokarstiques et l'abondance des massifs stalagmitiques exhumés confirment l'idée d'anciens systèmes karstiques démantelés. L'étude des cavités principales (grotte de Trabuc, évent de la Vernière) et de tronçons de galeries isolés, permet de proposer une évolution d'ensemble pour ces cavités. Le système majeur, formé en zone noyée profonde et peut-être sous couverture (marnes liasiques), a ensuite subi un comblement généralisé qui affecte toutes les cavités du secteur, quelle que soit leur altitude. L'incision des vallées provoque ensuite une réouverture du système. Certaines galeries sont réutilisées et partiellement vidées de leurs remplissages. Parallèlement, un nouveau système karstique se met en place, morphologiquement très différent du précédent, et en rapport avec la topographie commandée par l'enfoncement des Gardons. Au fur et à mesure de la dissection du massif, les galeries réutilisées sont de nouveau fossilisées et un important comblement calcitique s'y développe. Le recul des versants recoupe ces galeries et leurs remplissages affleurent en surface. Plusieurs traçages chimiques ont montré qu'il existe toujours une connexion hydrogéologique entre ces vieux tronçons et le niveau des circulations actuelles.

Mots-clés : géomorphologie, endokarst, paléokarst, remplissages, Mialet, Cévennes, Gard.
ABSTRACT : KARSTS AND PALEOKARSTS OF THE MIALET BASIN
(CÉVENNES, GARD, FRANCE) : FORMATION AND MORPHOLOGICAL EVOLUTION OF AN ANCIENT KARST CUT BY TOPOGRAPHICAL SURFACE.
The Mialet basin is a limestone massif of $62 \mathrm{~km}^{2}$ on the edge of the Cévennes Mountains, made of $400 \mathrm{~m}$ of limestones and dolomites. Two allochtonous rivers (Gardons)

cross and dissect it. Many caves are to be found at each level and their formation cannot be explained with today's topography. Surface karstic features (dolines) are rare but karstic fillings and many speleothems can be observed on the surface.

These deposits are former karst (paleokarst) cut by rivers incision. Recent karstic systems are organized differently but new subterranean circulations sometimes flow in old galleries and declog them.

Key-words : geomophology, endokarst, paleokarst, fillings, Mialet, Cévennes Mountains, Gard, France.

\section{INTRODUCTION}

Célèbre historiquement pour avoir été le lieu des guerres de religion (Camisards), la région de Mialet attire chaque année de nombreux touristes qui visitent en particulier la grotte de Trabuc. Cette cavité dont le développement atteint près de $10 \mathrm{~km}$ de galeries fait partie d'un massif calcaire mal connu des spéléologues, mais renfermant un karst original et bien développé. 


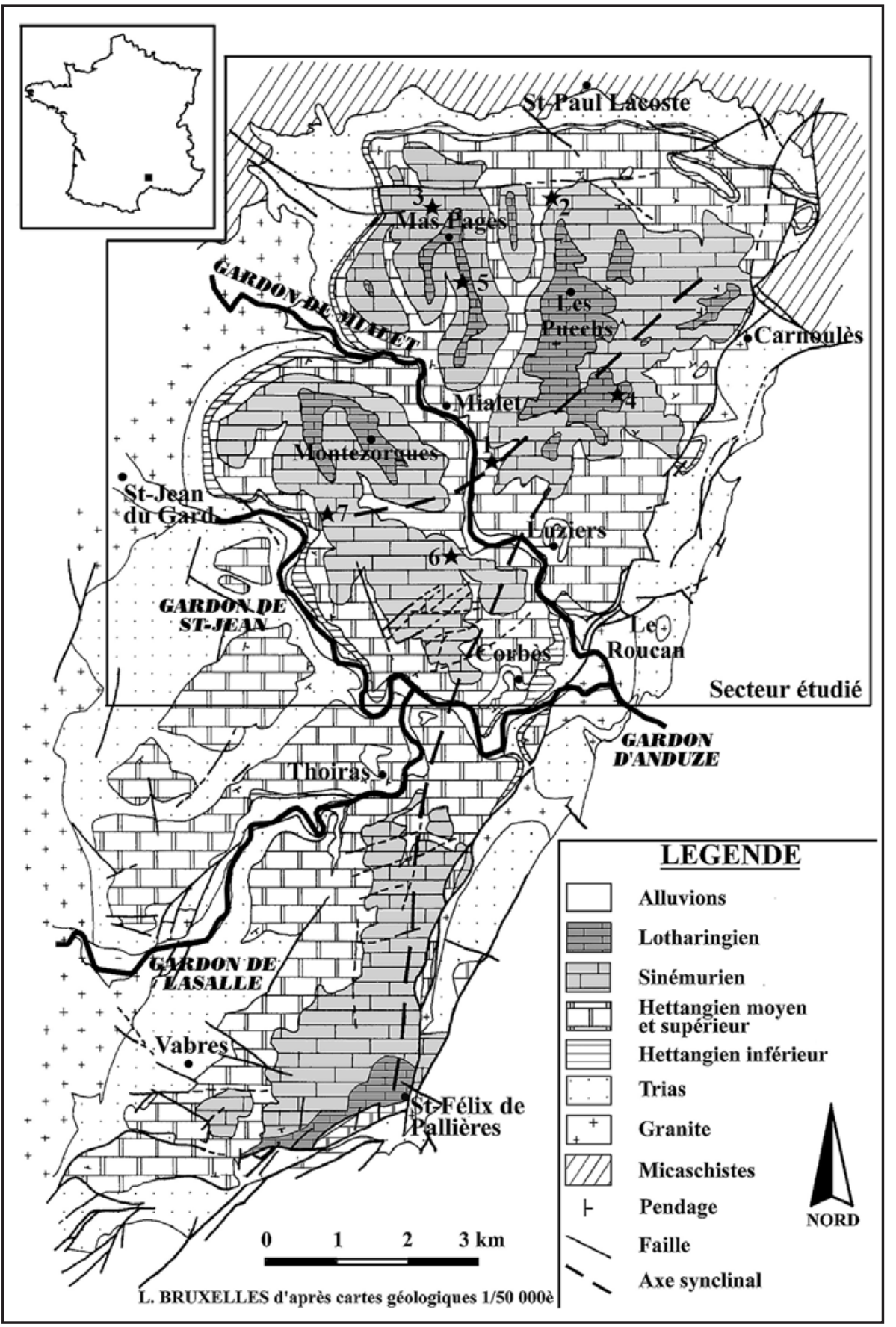

Figure 1 : Carte géologique du bassin de Mialet. La partie étudiée se situe au N du Gardon de St-Jean-du-Gard.

1- Grotte de Trabuc.

2- Event de la Vernière.

3. Aven du Calabrien.

4- Grottes de Camplone.

5- Grotte du Grand Issart.

6- Grotte de Valaurie.

7- Grotte de Rouville.

Geological map of Mialet basin.

The studied part is located north

of Gardon de St-Jean du Gard. supérieures de leur bassin versant. Les crues sont violentes et surprennent souvent par leur ampleur.

\section{APERÇU GÉOLOGIQUE}

Ce massif, constitué de dolomies et de calcaires du Lias, est encastré dans les Cévennes cristallines. Il est limité au $\mathrm{N}$ et à l'W par le contact avec le socle hercynien aux environs de St-Paul Lacoste et de St-Jean du Gard; au S et à l'E la limite suit la faille bordière NW du horst de Pallières (faisceau de la faille des Cévennes). Le secteur étudié ainsi délimité est formé d'une bande de terrains carbonatés d'une longueur approximative de $15 \mathrm{~km}$, pour 6 à $7 \mathrm{~km}$ de largeur et orientée NNE-SSW (fig. 1). Sa surface est voisine de $62 \mathrm{~km}^{2}$.

\section{A. Lithologie}

Durant le Trias et le Jurassique, des mouvements synsédimentaires sont à l'origine des variations de puissance et de faciès des formations sédimentaires entre la bordure et le centre du bassin, localisé approximativement au niveau de Mialet [Perrissol, 1990].

La série stratigraphique conservée comprend (de bas en haut) :

le socle hercynien formé de granite intrusif et de roches métamorphiques (micaschistes et gneiss). Sa partie supérieure, au voisinage de la surface d'aplanissement post-hercynienne, est souvent altérée sur une épaisseur qui peut atteindre plusieurs dizaines de mètres;

- le Trias transgressif sur le socle débute par un grès de base grossier, riche en éléments quartzeux fortement roulés (2 à $20 \mathrm{~mm}$ ) provenant du démantèlement des terrains primaires situés au $\mathrm{N}$. Il est surmonté par des formations saumâtres marno-gypseuses puis de plus en plus franchement marines (marno-gréso-dolomitiques). Les marnes dominent largement sur les cinquante derniers mètres, isolant (sauf contact tectonique local, Majencoule) les circulations et les cavités de la partie médiane du Trias de celles de la série calcaire sus-jacente;

l'Hettangien commence par $15 \mathrm{~m}$ de calcaires marneux gris constituant un important niveau à sources, surmontés par 100 à $150 \mathrm{~m}$ de dolomies grises massives à débit parallélépipédique (dolomie cubique). La majorité des cavités actives et quelques cavités fossiles de grande taille se situent dans ce niveau

- le Sinémurien est représenté par des calcaires argileux ou argilo-siliceux, gris-bleu, généralement noduleux, parfois riches en débris de crinoïdes. D'une puissance de 100 à $250 \mathrm{~m}$, la formation s'enrichit vers son sommet en silice sous forme de chailles irrégulièrement réparties Cet étage contient la plupart des grandes cavités de section importante; 


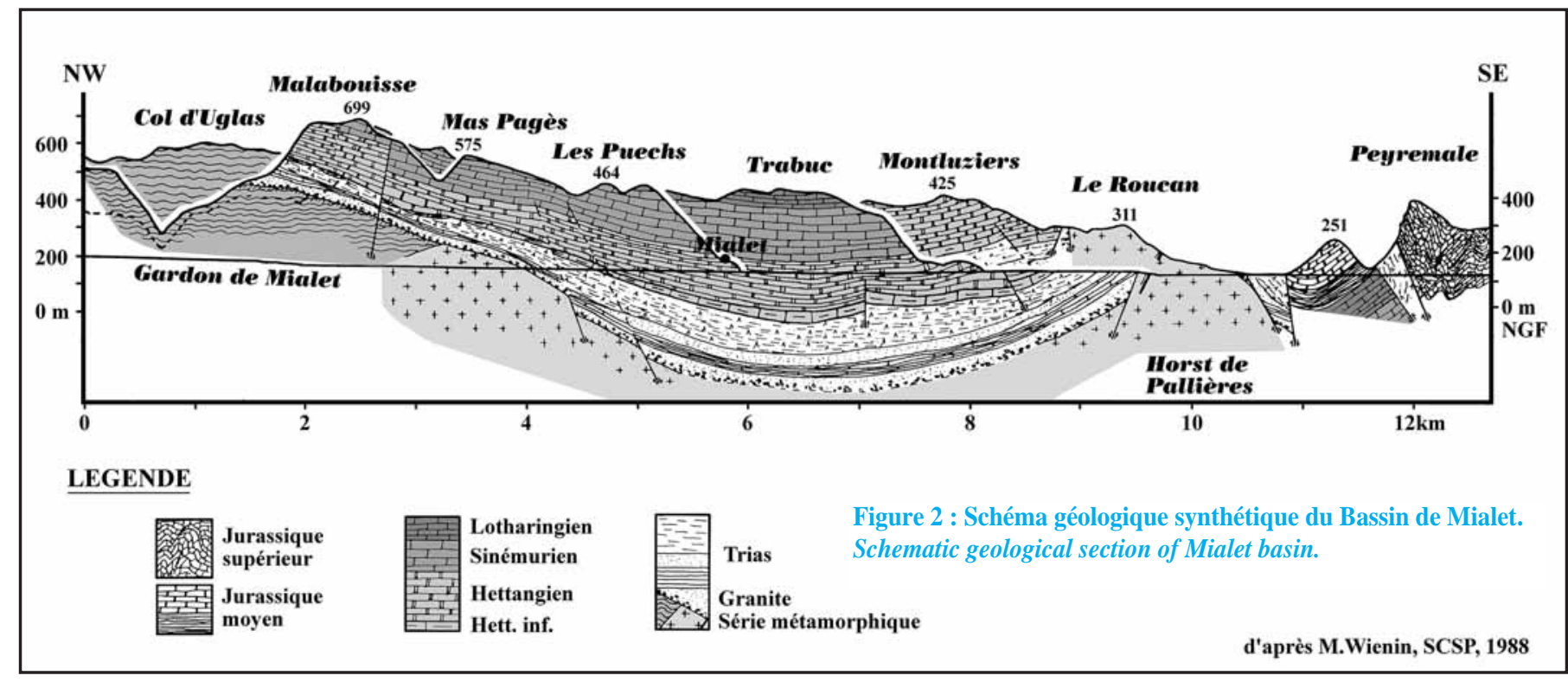

- le Carixien, calcaire gris bien lité, affleure autour de St-Félix avec un pendage au $\mathrm{S}$ limité par la faille du horst de Pallières qui piège quelques lambeaux de marnes domériennes.

\section{B. Structure}

Le Bassin de Mialet est un graben asymétrique lié au système de la faille des Cévennes (fig. 2). Il est limité au NW par la bordure cévenole. Celle-ci est abaissée par une série de failles normales. Au S, le contact avec le horst de Pallières se produit par l'intermédiaire d'un champ de failles minéralisées qui mettent en contact la dolomie hettangienne et le granite du Roucan. La série sédimentaire dessine une cuvette dont l'axe plus ou moins régulier a une orientation $\mathrm{N} 055^{\circ}$. Peu de failles affectent l'intérieur du bassin et les plus importantes sont situées sur la bordure.

On distingue globalement deux directions :

- les failles de direction cévenole (NNESSW);

- les failles E-W, plus rares, présentes au N et à l'extrême $S$ du secteur.

La distorsion horizontale du massif a provoqué des glissements bancs sur bancs au niveau des joints de strates. Ce phénomène, particulièrement net entre l'Hettangien et le Sinémurien (fonctionnement disharmonique), crée des discontinuités qui vont être exploitées par la dissolution. Dans le détail, l'étude tectonique du bassin de Mialet a montré que les flancs d'un graben sont divisés en "bandes" et en "panneaux" lorsque celui-ci subit la conjonction d'un mouvement d'enfoncement synclinal avec un coulissement senestre [Colas et Ruhland, 1982]. Ainsi, des fractures parallèles à l'axe du graben divisent ses flancs. Ils sont composés de "bandes" de blocs basculés d'une dizaine de mètres de largeur, assez fortement fractu- rées et limitées de part et d'autre par deux diaclases (fig. 3). Ces structures isolent des "panneaux" d'extension plus importante (100 à 200 m) à l'intérieur desquels le pendage est homogène. Cette disposition joue un grand rôle dans l'organisation du karst et la morphologie des principaux conduits. La topographie de la grotte de Trabuc est à ce niveau tout à fait représentative (fig. 4 et 5).

\section{IF KARST DU BASSIN DE MIALET}

Les formes exokarstiques sont rares dans ce secteur. Les dolines sont quasi-inexistantes, et les lapiaz sont réduits à quelques petites tables dispersées. Il n'existe pas de reculée karstique bien marquée, et l'on ne connait aucune perte importante. En fait, les indices externes qui permettent de soupçonner l'existence d'un endokarst aussi développé sont presque absents. Nous avons pourtant recensé et visité plus de 200 cavités, dont certaines n'ont rien à envier, par leur section et leur développement, aux cavités d'autres massifs karstiques plus célèbres.

\section{A. Les cavités principales}

\section{La grotte de Trabuc}

Cette cavité est le réseau majeur du système (fig. 4). Son développement atteint $10 \mathrm{~km}$ pour un dénivelé total de $205 \mathrm{~m}$ [SCSP, 1988]. Les galeries principales sont sur le flanc SE du synclinal, mais les circulations actuelles se font au niveau d'une zone broyée, située sur l'axe de la gouttière (faille axiale). La majeure partie des galeries sont

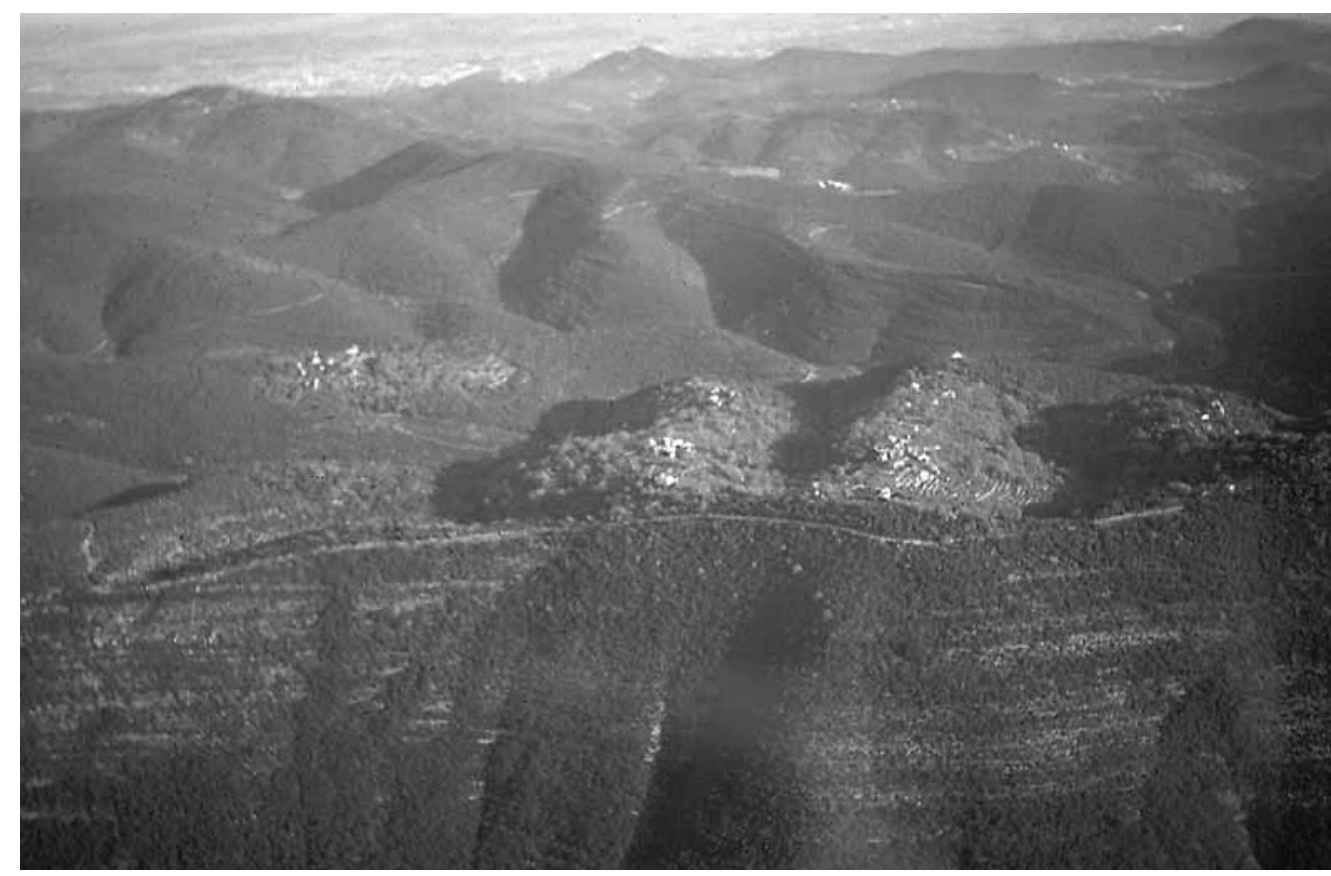

Photo 1 : Vue d'ensemble vers le S du massif de Mialet. Au premier plan le hameau des Puechs (cliché L.B.). General view of Mialet basin. In the foreground, hamlet of Les Puechs. 
Photo 2 : Grotte de Trabuc : sommets de coupoles de plafond dans la Galerie de la

fourche. L'effondrement des strates calcaires n'est pas assez important pour effacer toutes les formes de creusement en régime noyé (cliché L.B.). Tops of roof coupolas into the "Galerie de la Fourche". Collapse of limestone layers is not important enough to mask the flooded karst morphologies.

fossiles, de grosse section (10 x $10 \mathrm{~m}$ en moyenne) et se localisent pour la plupart au contact entre la dolomie de l'Hettangien et le Sinémurien calcaire.

On distingue deux morphologies distinctes :

- des galeries présentant de nombreuses formes de creusement en régime noyé; ce sont souvent des sections développées dans la dolomie hettangienne, très massive, et donc favorable à la conservation de ces formes (Vieux Trabuc, Lac Nord, Anniversaire);

- des galeries à section “carrée" : d'une hauteur dépassant fréquemment $30 \mathrm{~m}$, ces galeries se sont formées au contact Hettangien-Sinémurien. Elles ont évolué par

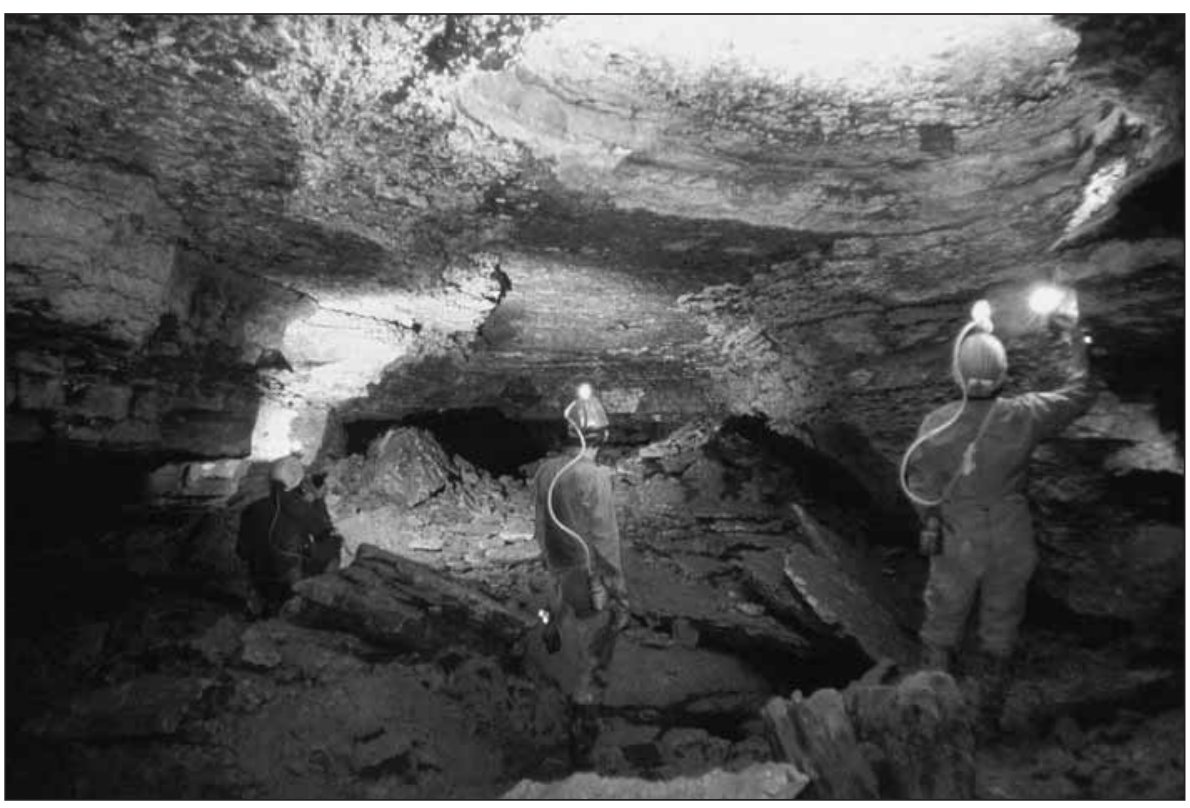

effondrement-dissolution le long des "bandes" tectoniques NNE-SSW décrites plus haut et dont le rôle morphologique est indéniable. Le plafond stratiforme résulte de la chute des bancs de calcaire sinémurien, noduleux et au litage plus marqué à ce niveau, qui s'accumulent sur un abondant matériel détritique. Certaines galeries à section quadrangulaire présentent encore à
Figure 3 : Bloc diagramme montrant la fracturation du $\mathrm{S}$ du graben :

a) en bandes et en panneaux. b) division des bandes en "lanières" [Colas et Ruhland, 1982]. Block diagram showing the fracturing of the south part of the graben :

a) into bands and larger segments. b) bands divided in "lanières" [Colas et Ruhland, 1982].

Tableau 1 : Résultat des datations U/Th

d'échantillons de spéléothèmes

(Y. Quinif, CERAK,

Mons). U/Th results (Y. Quinif, CERAK, Mons).

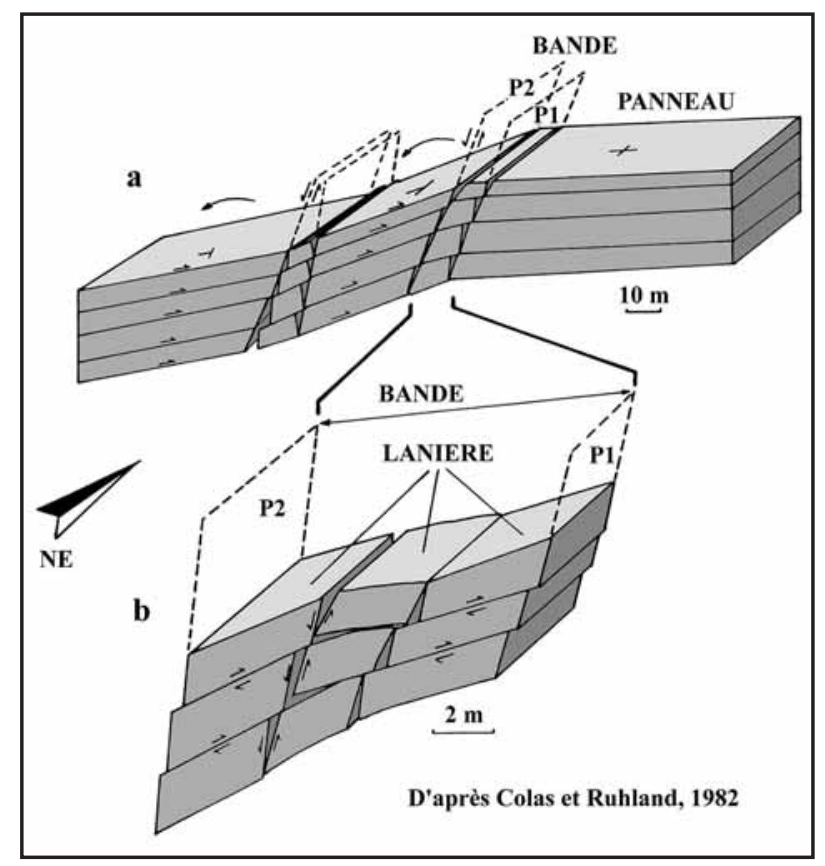

D'après Colas et Ruhland, 1982

\begin{tabular}{|c|c|c|c|c|c|c|}
\hline Echantillons & (U) ppm & $\begin{array}{l}234 \mathrm{U} / \\
238 \mathrm{U}\end{array}$ & $\begin{array}{l}\text { 230Th } \\
\text { /234U }\end{array}$ & $\begin{array}{l}\text { 230Th } \\
\text { /232Th }\end{array}$ & $\begin{array}{c}(234 \mathrm{U} \\
/ 238 \mathrm{U}) \mathrm{t}=0\end{array}$ & Age \\
\hline $\begin{array}{c}\text { Galerie de } \\
\text { l'Anniversaire } \\
\text { (Trabuc) }\end{array}$ & $\begin{array}{r}0,192 \\
\pm 0,004\end{array}$ & $\begin{array}{r}1,056 \\
\pm 0,025\end{array}$ & $\begin{array}{r}1,092 \\
\pm 0,097\end{array}$ & $21 \pm 8$ & ---------- & $>400000$ \\
\hline $\begin{array}{l}\text { Paléokarst route } \\
\text { des Puechs (Mialet) }\end{array}$ & $\begin{array}{r}0,110 \\
\pm 0,004\end{array}$ & $\begin{array}{r}0,990 \\
\pm 0,044\end{array}$ & $\begin{array}{r}0,964 \\
\pm 0,080\end{array}$ & $47 \pm 29$ & ---------- & $>400000$ \\
\hline
\end{tabular}

la voûte le sommet d'importantes coupoles, attestant leur formation en régime noyé (photo 2).

Cette cavité présente un comblement important d'éléments détritiques, constitués de matériel autochtone (argile et galets de chailles), et d'éléments clastiques (dalles calcaires). La progression dans la cavité donne l'impression d'une succession de zones fortement colmatées et de secteurs réutilisés ou déblayés par soutirage, en connexion avec les niveaux inférieurs actifs.

Lorsqu'on s'éloigne des points de soutirage, on bute sur des galeries encore totalement comblées ou en voie de décolmatage (galerie de l'Anniversaire).

Le concrétionnement est très abondant tout au long de la cavité et forme localement d'imposants massifs stalagmitiques. La datation (Y. Quinif, CERAK) d'un petit plancher stalagmitique reposant sur le remplissage détritique de la galerie de l'Anniversaire donne un âge supérieur à 400000 ans (fig. 6 et tableau 1).

Le niveau actif pérenne de Trabuc se présente comme un ensemble de petites conduites forcées et de diaclases actives surtout en période de crue. Morphologiquement très différent, il est connecté en divers points aux vastes galeries. Lors des crues, la saturation de ce niveau provoque des débordements violents et l'eau réutilise temporairement certains tronçons de galeries supérieures. À l'étiage, de nombreux siphons constituent des regards sur la zone noyée, siège des circulations plus profondes. Un de ces siphons (siphon Golenvaux) a permis de progresser sur plusieurs centaines de mètres dans un conduit situé à plus de $40 \mathrm{~m}$ sous le niveau du Gardon. 


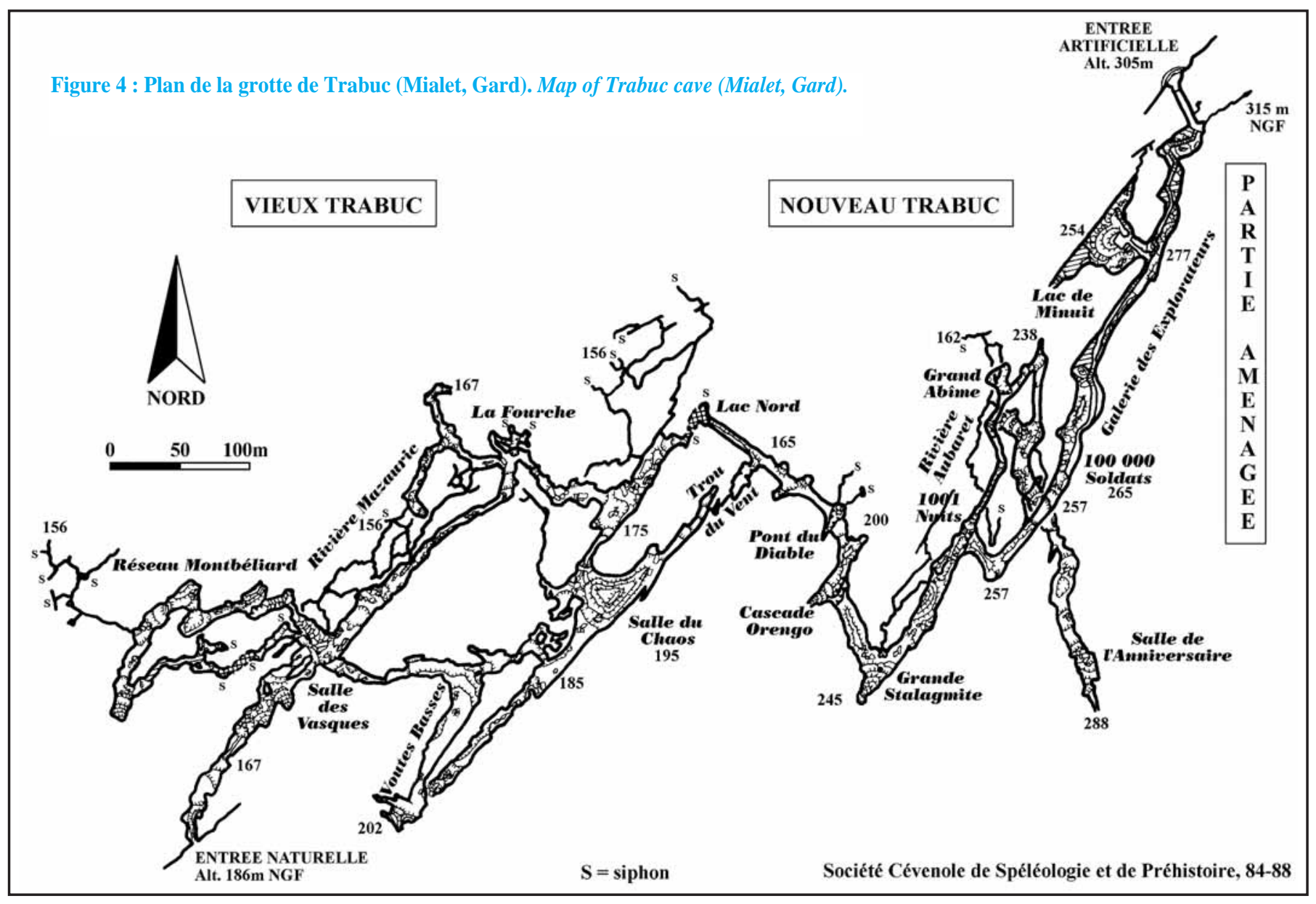

\section{L'évent de la Vernière}

Découvert récemment, l'évent de la Vernière est une cavité temporairement active constituée d'une galerie unique, de grande taille. Creusée dans les calcaires du Sinémurien, elle ressemble plutôt à une haute galerie rappelant celles de Trabuc, dont la largeur varie entre 3 et $10 \mathrm{~m}$ pour une hauteur dépassant parfois $30 \mathrm{~m}$ (photo 3 ). On retrouve par endroits la section carrée caractéristique des galeries de Trabuc et son mode de formation découle sans doute du même processus.

Ici aussi, le comblement est important et plusieurs massifs stalagmitiques obstruent totalement la galerie. Seule l'activité temporaire de cette cavité a permis un recreusement du remplissage détritique présent sous les massifs de calcite. On descend alors sur plus de $30 \mathrm{~m}$ à travers des sédiments (calcite, argile, sable, galets de chailles) pour accéder à la rivière pérenne. Il est aussi possible de traverser horizontalement le remplissage, recreusé sous le massif de calcite, pour déboucher $50 \mathrm{~m}$ plus loin dans la suite de la cavité. On se trouve alors exactement de l'autre côté du massif stalagmitique. La galerie principale se termine sur un autre massif de concrétion qui l'obstrue sur toute sa hauteur $(35 \mathrm{~m})$. La présence de racines en ce point annonce la proximité de la surface (fig. 7).

\section{B. les cavités de deux ième ordre}

Outre ces deux réseaux majeurs, d'autres cavités méritent d'être décrites ici, bien que de taille plus modeste.

\section{Rive gauche du Gardon de Mialet}

Les avens sont rares dans ce secteur et résultent soit d'un phénomène de détente des versants, soit de l'effondrement de la voûte d'une cavité sous-jacente.

- L'aven du Calabrien : Cette cavité, dont l'orifice mesure près de $30 \mathrm{~m}$ de diamètre, relève du second cas. Peu profonde, elle correspond à une ancienne salle, assez vaste, aujourd'hui en grande partie comblée par les blocs éboulés. Latéralement, le franchissement d'un massif stalagmitique, aujourd'hui à l'air libre, permet d'accéder à une courte galerie de nouveau obstruée par la calcite. Cette cavité à fait l'objet d'une coloration

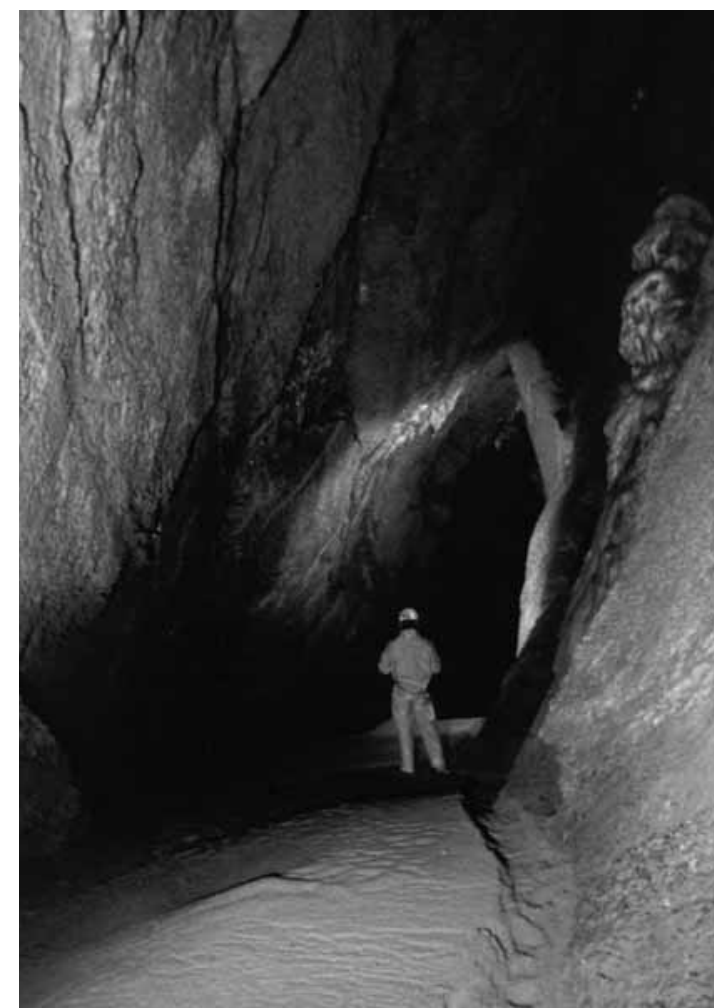

Photo 3 : Galerie principale de l'Event de la Vernière. Le remplissage détritique et chimique occupe plus des 2/3 de la hauteur initiale (cliché $\mathrm{S}$. Bruxelles).

Main gallery of "Event de la Vernière".

Detrital and chemical filling reduce height of 2/3. 


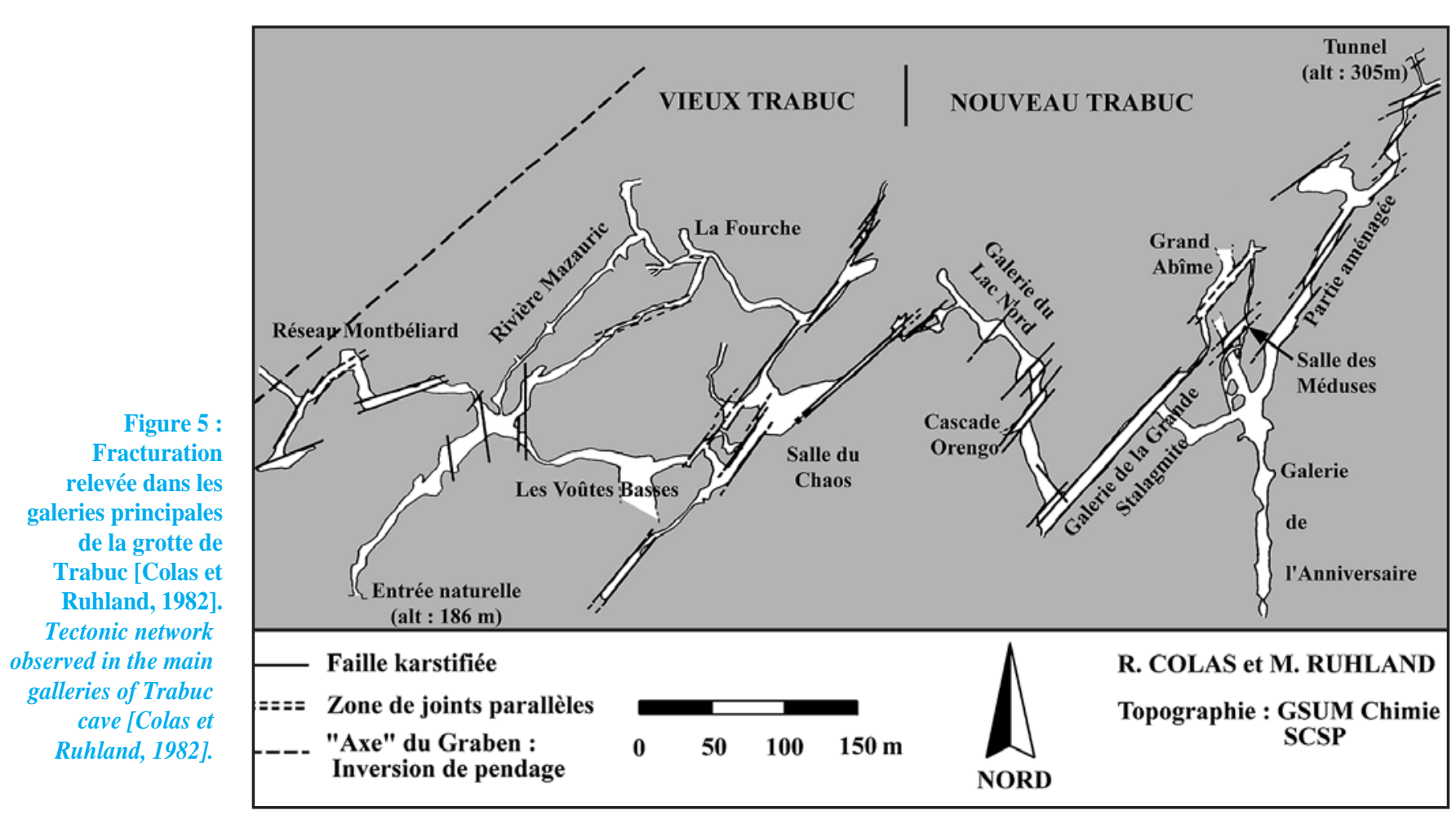

[Bruxelles, 1994]. Le colorant déversé au pied de la coulée terminale s'est infiltré à travers le remplissage argileux. Il est ressorti 5 jours plus tard à l'exsurgence du Moulenc, située $353 \mathrm{~m}$ plus bas, prouvant ainsi que des connexions hydrogéologiques existent toujours entre ces vieilles cavités décapitées et les niveaux actifs.

- Les grottes de Camplone : Elles sont situées au sommet d'une colline et se développent dans le Lotharingien (Sinémurien sup.). Les deux entrées se trouvent à quelques mètres l'une de l'autre, au sommet d'un massif stalagmitique qui sépare désormais la galerie en deux tronçons distincts (fig. 8). Leur section reste importante malgré l'amoncellement de blocs détachés de la voûte.

Les morphologies du creusement initial ont disparu laissant place à une section carrée classique. Au bout de $50 \mathrm{~m}$, la plus grande des deux grottes bute sur une trémie de gros blocs située très près de la surface. Sur le versant, dans l'axe de la cavité et quelques mètres en contrebas, une importante quantité de calcite matérialise l' ancien prolongement de la galerie.

- La grotte du Grand Issart : La présence en surface d'un important massif stalagmitique non loin de la crête permet la découverte de cette cavité. Elle est formée d'une galerie unique de plus de $200 \mathrm{~m}$ de longueur. Elle atteint $8 \mathrm{~m}$ de section mais présente des rétrécissements au sommet d'imposants massifs stalagmitiques. Son tracé rectiligne implique que le terminus est proche du versant opposé à l'entrée. Ceci a été confirmé par la localisation en surface d'un massif stalagmitique exhumé. Cette grotte traverse donc de part en part la crête (fig. 9) tout en conservant, malgré sa position sommitale (300 $\mathrm{m}$ au-dessus des niveaux actifs), de belles formes de creusement (coupoles de

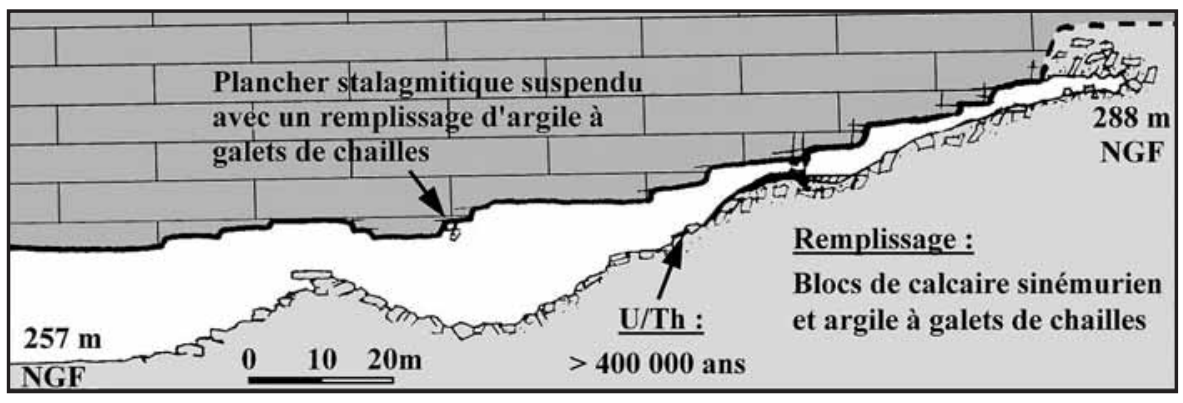

Figure 6 : Coupe schématique de la terminaison de la galerie de l'Anniversaire dans la grotte de Trabuc. La base est creusée au contact Hettangien-Sinémurien ( $265 \mathrm{~m}$ NGF). Le plancher daté repose sur le remplissage partiellement déblayé ("front" de décolmatage).

Section of the "Galerie de l'Anniversaire" in the Trabuc cave ending. The dated flowstone is situated on the detritial filling. plafond, coups de gouge) et un remplissage détritique en cours d'étude (galets de chailles et argile).

\section{Rive droite du Gardon de Mialet}

Les massifs de Corbès et de Montezorgues renferment quelques cavités qui sont comparables en plusieurs points avec celles présentées plus haut.

- La grotte de Valaurie : Située au N de Corbès, elle s'ouvre à $275 \mathrm{~m}$ d'altitude, non loin d'affleurements de calcite. Elle est constituée d'une galerie principale d'une centaine de mètres développée au contact Hettangien-Sinémurien. Sa morphologie ainsi que sa section en font une cavité très semblable aux galeries principales de Trabuc. Le remplissage détritique puis chimique comble en partie ce conduit, mais quelques indices montrent qu'elle a été intégralement colmatée.

- La grotte de Rouville : Dominant le Gardon de St-Jean, cette cavité est une vaste galerie à section carrée qui débouche à flanc de versant, à 352 m d'altitude. En pente régulière vers l'E, elle est conforme au pendage local des niveaux calcaires du Sinémurien. Sa section, de $10 \mathrm{~m}$ x $8 \mathrm{~m}$ à l'entrée, est constante pendant une cinquantaine de mètres. Ensuite, elle s'amenuise régulièrement et devient impénétrable à $110 \mathrm{~m}$ de l'entrée lorsque le sommet du remplissage atteint la voûte Dans ces deux cavités, des expériences de coloration ont mis en évidence leur drainage par des petites sources. 


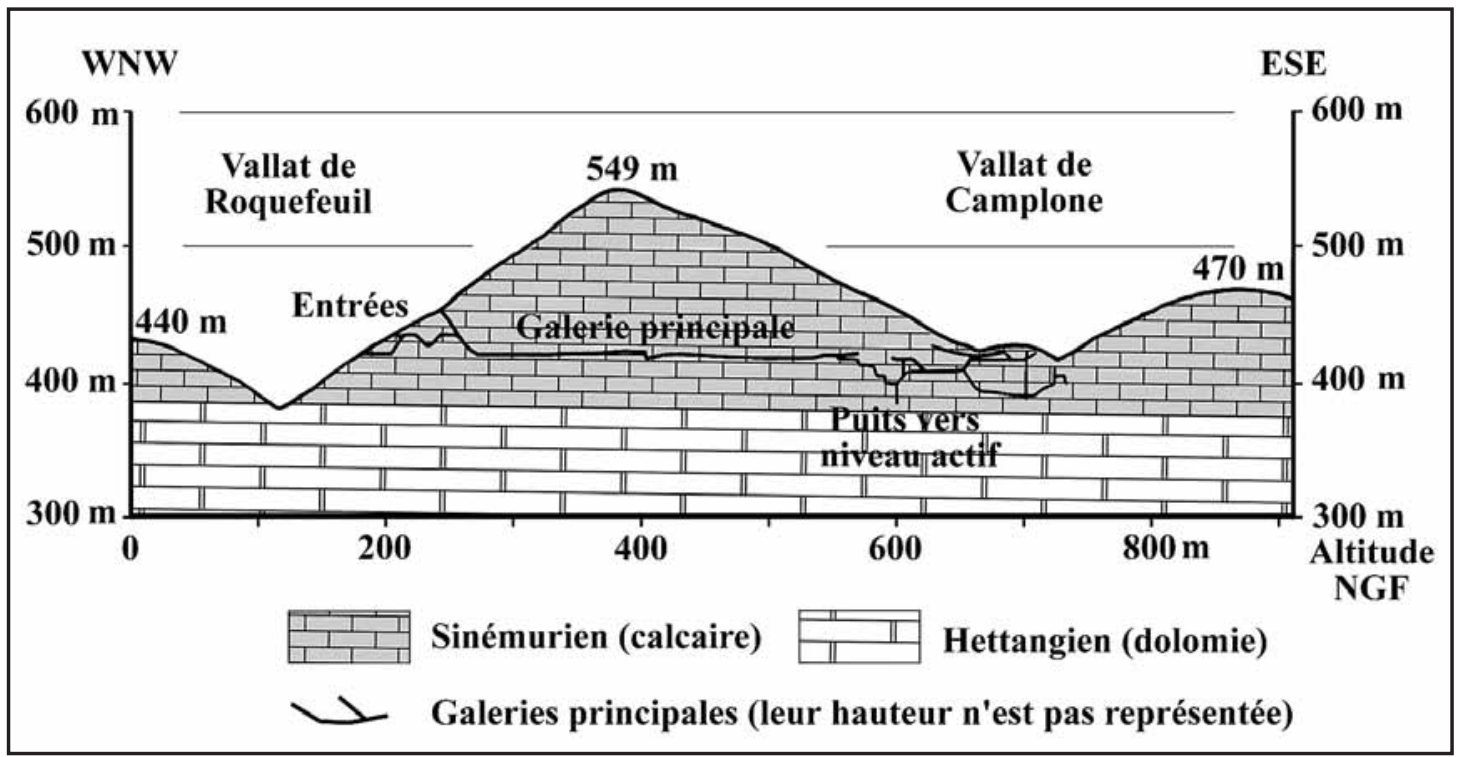

Figure 7 : Coupe

topographique le long de l'axe principal de l'évent de la Vernière (Mialet).

Section along the main gallery of the "Event de la Vernière".

Le colorant, poussé par des écoulements temporaires, a traversé le remplissage de ces grottes avant d'être restitué par des drains karstiques d'une tout autre facture.

\section{Les remplissages endokarstiques ex humés}

Ils atteignent une fréquence et une ampleur peu commune, sans doute en relation avec l'intense karstification du secteur, mais aussi avec son importante dissection.

Cependant, la cartographie de ces éléments ne permet pas, comme cela a été fait sur le Causse de Blandas (Gard), de retrouver sur une grande distance le tracé des paléo-réseaux karstiques [Ambert et al., 1978].

Nous pouvons distinguer deux types de remplissages endokarstiques exhumés qui matérialisent en réalité deux stades de l'évolution de ces cavités.

\section{Les poches de sédiments détritiques}

Difficiles à remarquer, elles sont révélées lors de travaux (ouverture d'une piste). Il en existe une dizaine sur la piste joignant le Col d'Uglas et le Mas Pagès, à une altitude comprise entre 500 et $600 \mathrm{~m}$. Elles correspondent à d'anciens conduits karstiques aujourd'hui décapités, dont la largeur atteignait plusieurs mètres.

Ces galeries sont totalement colmatées par des sédiments détritiques constitués d'argile et de galets de taille centimétrique à pluri-centimétrique nettement lités (photo 4).

Une rapide étude de la fraction grossière nous a permis de recenser du matériel autochtone, constitué exclusivement de fragments de chailles issus des calcaires du Sinémurien et du Lotharingien.

\section{Les édifices stalagmitiques}

Très nombreux, ils sont présents à toutes les altitudes, depuis les crêtes où ils sont fréquents, jusqu'à la base des versants réglés (photo 5). On y reconnaît parfaitement les structures de la calcite et les détails les plus fins des concrétionnements endokarstiques (stalactites, stalagmites, micro-gours). Ces édifices se localisent aussi bien dans la dolomie hettangienne que dans les niveaux calcaires. Leur mise au jour est due au recoupement d'une galerie par l'enfoncement de la surface topographique. Selon la taille et l'orientation de cette galerie, l'affleurement de calcite peut former un redan rocheux atteignant plusieurs mètres de largeur et de hauteur, pour une longueur pouvant dépasser une centaine de mètres, comme sur la colline de Teizière. La datation de ce massif stalagmitique a donné, sans grande surprise d'ailleurs, un âge supérieur à 400000 ans (tableau 1). Parfois, le massif stalagmitique surmonte une formation détritique semblable à celle trouvée dans les poches précédemment décrites. Il correspond alors à un stade intermédiaire illustrant bien l'histoire polyphasée de ces karsts.

\section{FORMATION ET EVOLUTION DU KARST}

De nombreux éléments, tant en surface (lapiaz démantelés) qu'en profondeur (cavités tronquées), indiquent sans ambiguité le caractère résiduel de ce karst. En aucun cas, la topographie actuelle ne permet d'expliquer la genèse et la localisation de ces réseaux.

\section{A. Mise en place du karst}

À l'origine, sous une topographie différente, ces portions de galeries étaient

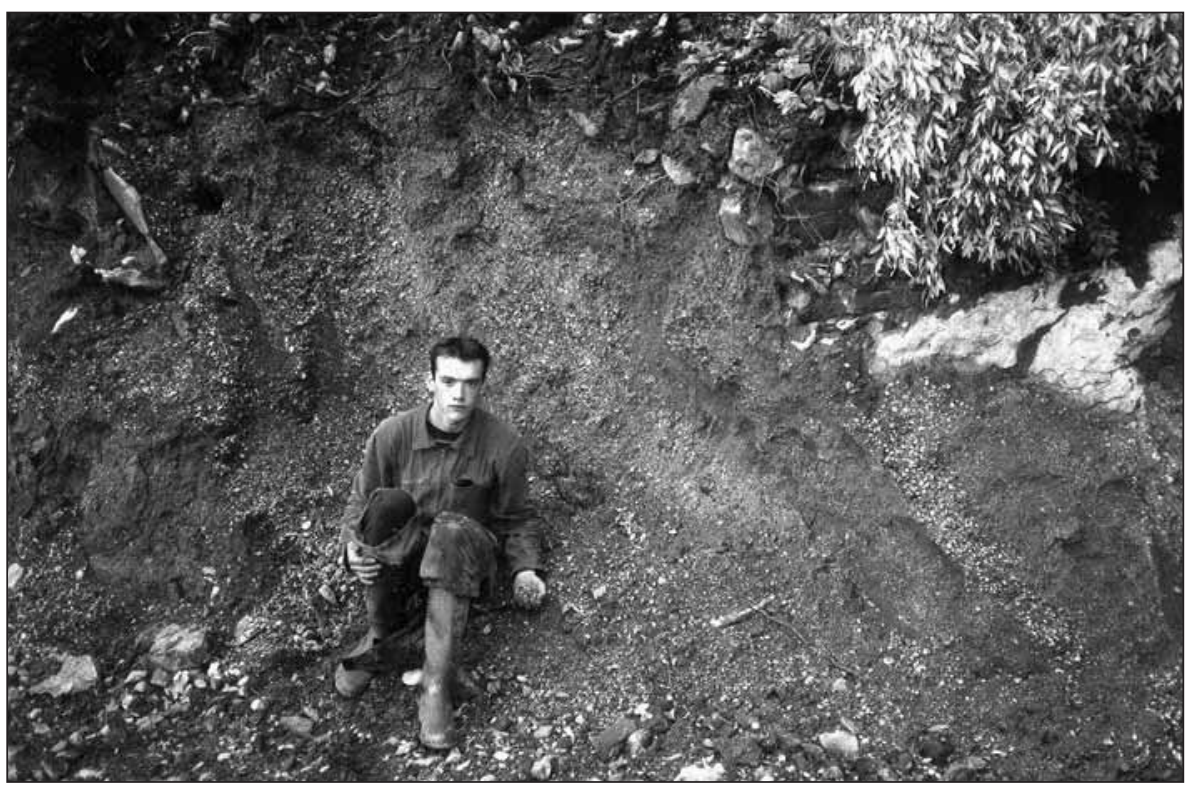

Photo 4 : Poche d'argile et de galets de chailles à l'E du Mas Pagès. Le litage est nettement visible à droite du personnage (cliché L.B.). Filled pocket with detrital sediment. 
Figure 8 : Topographie des grottes de Camplone

(Mialet). La surface

topographique recoupe plusieurs fois cette ancienne

galerie actuellement en position sommitale. Map and section

of "Grottes de Camplone".

The topographical surface cut this gallery several times.

connectées et formaient un ou plusieurs grands réseaux karstiques noyés. L'étude de la répartition des galeries [Bruxelles, 1994] ne permet pas d'isoler une ou des altitudes préférentielles corrélatives d'anciens niveaux de base liés à différents stades d'enfoncement du Gardon.

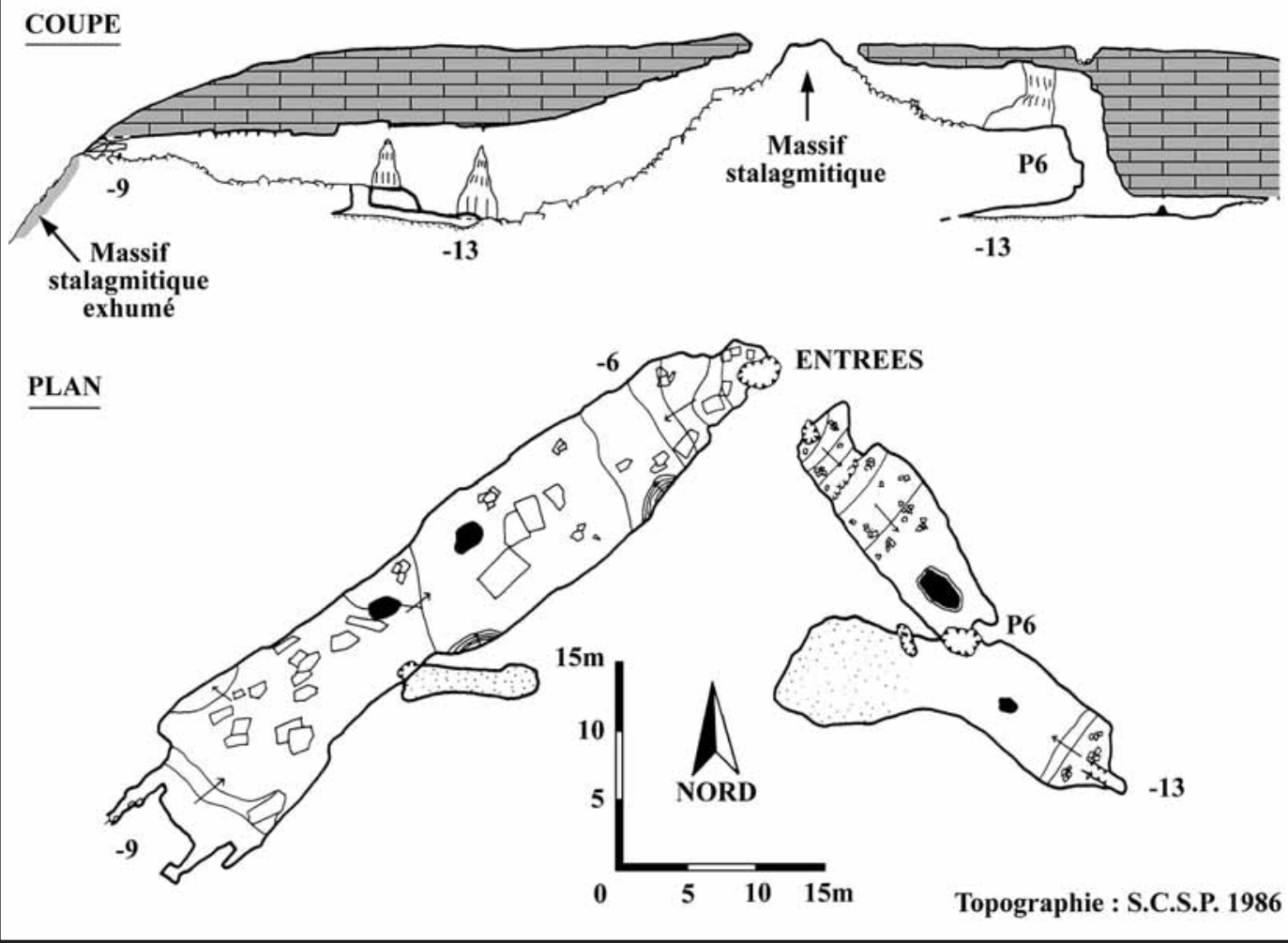

L'apparente corrélation entre

la situation altitudinale de certaines cavités n'est qu'illusoire. En fait, le développement de ce karst semble plutôt s'être opéré en zone noyée profonde, le long des discontinuités lithologiques en suivant les faciès les plus favorables à la karstification (essentiellement contact Hettangien-Sinémurien), et guidé par les divers types de fractures. L'indifférence de la karstification vis-à-vis de la structure générale confirme cette idée de karst profond noyée. D'ailleurs, les galeries principales de la grotte de Trabuc, comme celle de l'évent de la Vernière ne se localisent pas au fond du synclinal mais sur les flancs et à des niveaux très variables.
La série du Jurassique inférieur est surmontée stratigraphiquement par une très forte épaisseur de marnes du DomérienToarcien. La genèse de ce karst noyé sous couverture est une hypothèse valable qui répond parfaitement aux caractéristiques de l'ensemble de ces cavités et à leur organisation.

\section{B. Colmatage généralisé des cavités}

Par la suite, selon des modalités qui sont à préciser, ce karst encore noyé a subi un comblement quasi-total par des sédiments

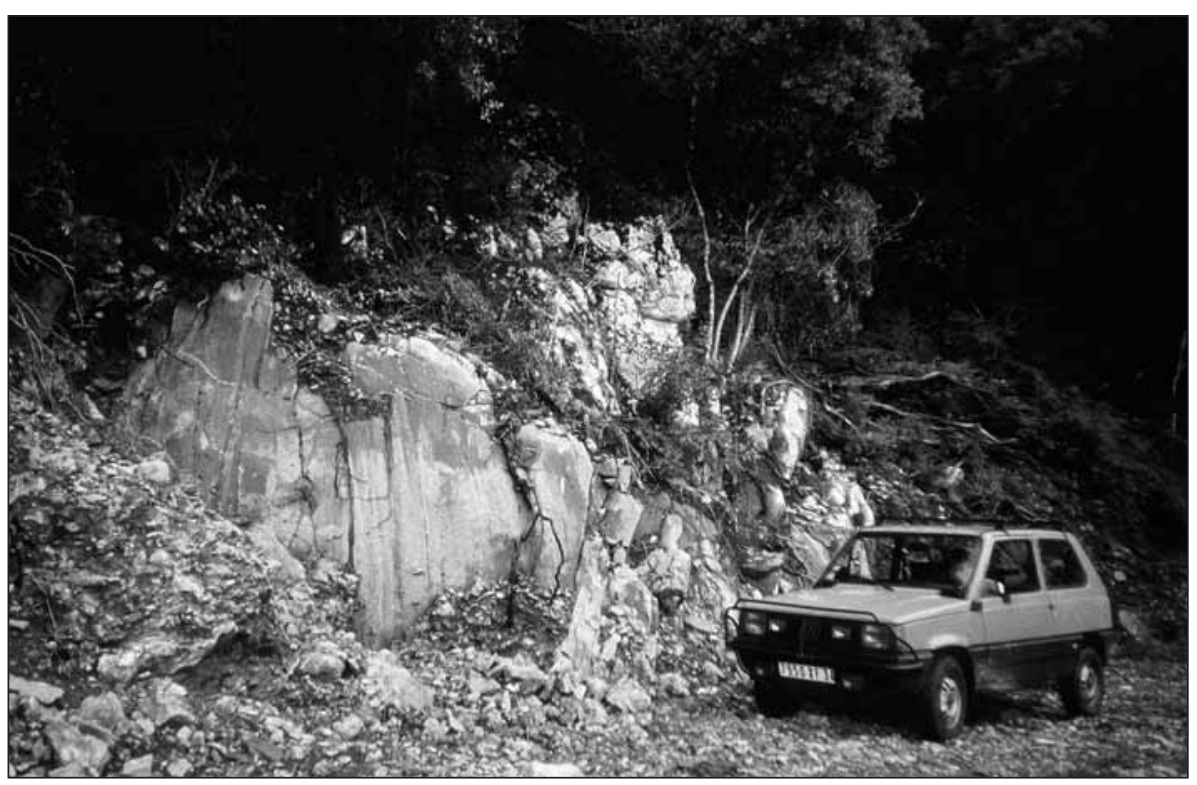

Photo 5 : Ce massif stalagmitique exhumé, situé à proximité du Mas Pagès, matérialise la prolongation d'une ancienne galerie actuellement recoupée par le versant (cliché

S. Bruxelles). These speleothems exhumed near Mas Pagès show the floor of an old gallery.

constitués d'éléments autochtones très semblables (argile et galets de chailles), et cela dans toutes les grandes cavités du bassin de Mialet, quelle que soit leur altitude. Dans la grotte de Trabuc, par exemple, de nombreuses galeries de plusieurs dizaines de mètres de hauteur conservent les témoins d'un colmatage complet (Cascade Orengo, Grande Stalagmite, Salle des Méduses). Cette homogénéité des sédiments endokarstiques dans les cavités du bassin de Mialet semble, elle aussi, aller dans le sens d'une histoire peu diversifiée avec développement des réseaux en profondeur puis leur colmatage général. Plusieurs causes peuvent être invoquées pour expliquer ce phénomène : variation morphologique ou changement climatique; mais seule une subsidence généralisée de ce petit bassin peut justifier son comblement total par du matériel homogène local sur une aussi grande dénivellation. Par comparaison avec le bassin d'Alès tout proche, le bassin de Mialet a dû être également subsident à l'Oligocène. L'essentiel de ce karst, aujourd'hui décolmaté, était donc formé dès l'Oligocène.

\section{Démantèlement et réorganisation karstique}

Quelques alluvions siliceuses (schistes, micaschistes) subsistent sur des lambeaux de plateaux constituant les point hauts du massif. Elles témoignent de la mise en place du réseau hydrographique provenant des Cévennes cristallines partiellement dénudées de leur couverture sédimentaire. La présence d'alluvions siliceuses dans les remplissages 


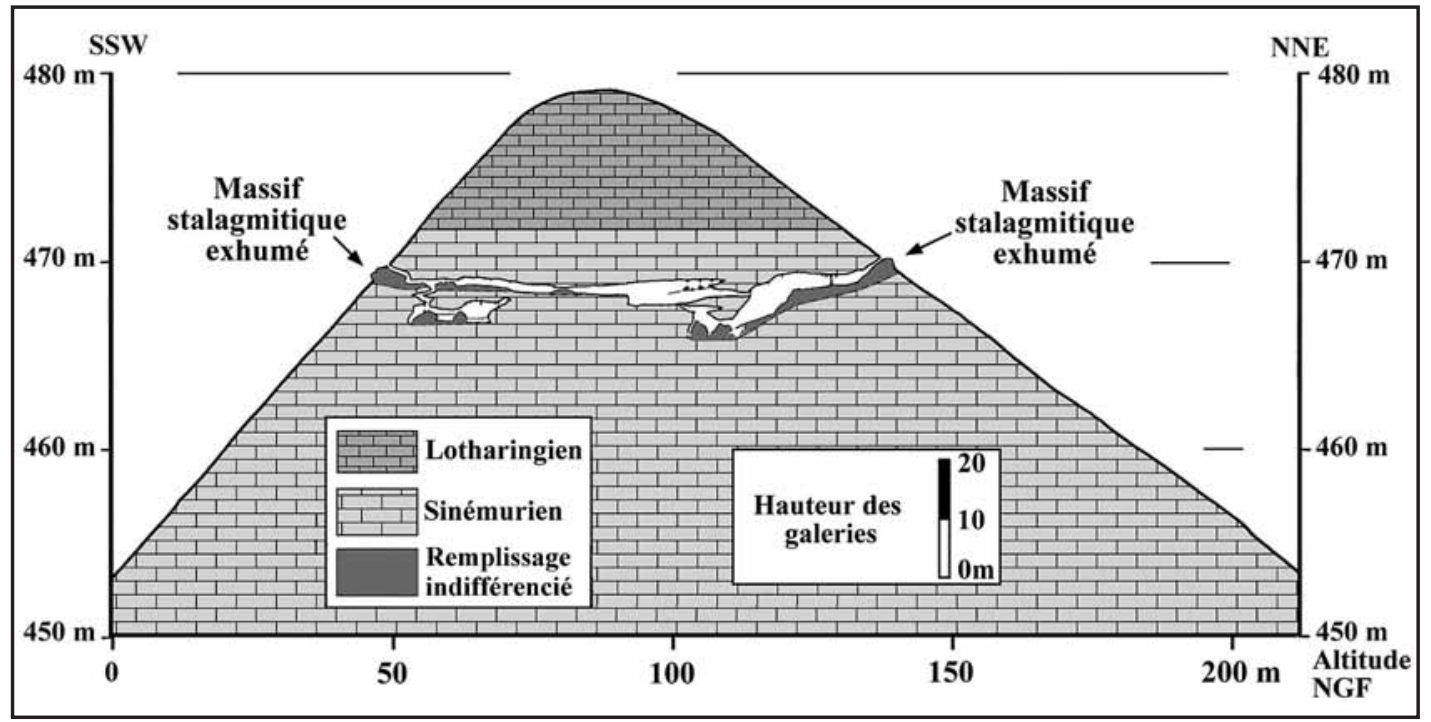

Figure 9 : Coupe

topographique le long

de l'axe principal de

la grotte du Grand Issart.

D'imposants massifs

stalagmitiques

matérialisent l'ancien

prolongement

de cette cavité.

Cross section along the main gallery of the "Grotte

du Grand Issart".

On the slopes, speleothems

show the floor of the

ancient cave passages.

karstiques serait inévitable si la karstification du massif était postérieure à leur dépôt. Leur absence dans les remplissages décrits plus haut montre que le karst préexistait et était déjà entièrement colmaté.

L'incision des Gardons a permis une réouverture du système. Une nouvelle organisation karstique, très différente de la première, se met en place. L'influence de la structure d'ensemble est alors plus marquée et le synclinal fait office de collecteur des eaux souterraines en rapport avec le niveau de base commandé par l'enfoncement des Gardons. Sur les flancs, les différentes discontinuités sont exploitées par les circulations karstiques dans le sens du gradient hydraulique, en direction des rivières principales ou des cours d'eau affluents (Amous, Roquefeuil, Séboullière). Ces écoulements, plus locaux, sont drainés par des nouveaux conduits karstiques de petite taille, souvent impénétrables et se présentent sous la forme de petites conduites forcées associées à des diaclases à peine élargies. Les circulations récentes vont recouper et réutiliser des tronçons d'anciens conduits karstiques, plus vastes, mais en grande partie comblés. Cette nouvelle activité va partiellement les décolmater, soit par réutilisation directe (évent de la Vernière), soit par soutirage (galerie de la Grande Stalagmite et le Grand Abîme à Trabuc). Elle affecte essentiellement les cavités situées à proximité de ces nouveaux drains. Les galeries les plus éloignées des nouveaux axes de drainage ne subiront qu'un soutirage modéré ou une évacuation partielle du remplissage par érosion régressive, sans avoir nécessairement connu de réutilisation directe (galerie de l'Anniversaire à Trabuc).

Lors du décolmatage de ces galeries, désormais hors d'eau, les parois sont affectées d'un phénomène de détente. Des pans entiers se détachent et s'effondrent au sol (photo 6). La voûte, moins soutenue s'effondre à son tour et confère à ces galeries cette section dite “carrée" (photo 7). Ce processus d'effondrement peut se poursuivre sur plusieurs dizaines de mètres (au moins $60 \mathrm{~m}$ dans la partie aménagée de Trabuc) rappelant la formation, à une échelle moindre, des cheminées de soutirage [Renault, 1993].

Au fur et à mesure de l'enfoncement du réseau hydrographique, le karst subit un démantèlement croissant divisant le massif en petites unités calcaires de plus en plus indépendantes. Les cavités réutilisées se fossilisent de nouveau. Un abondant concrétionnement se développe et apparaît au jour lorsque la galerie est décapitée. Quelques massifs stalagmitiques se forment au gré des infiltrations dans l'ensemble de la cavité. D'autres sont liés à la proximité de la surface. La détente des versants favorise leur développement dans le secteur d'entrée et le recul des versants permet une progradation de ces massifs vers l'intérieur de la cavité. En surface, on peut suivre un long affleurement de calcite de plusieurs dizaines de mètres jusqu'à l'orifice de la cavité. Parfois, une partie de l'ancien remplissage détritique, conservée sous la calcite, affleure sous le massif de concrétion. Il libère sur le versant de très nombreux galets ovoïdes de chailles (massif stalagmitique de la piste du Boisset). D'autres galeries, non réutilisées, restent totalement comblées et forment ces poches de sédiments détritiques mises au jour par le recul des versants.

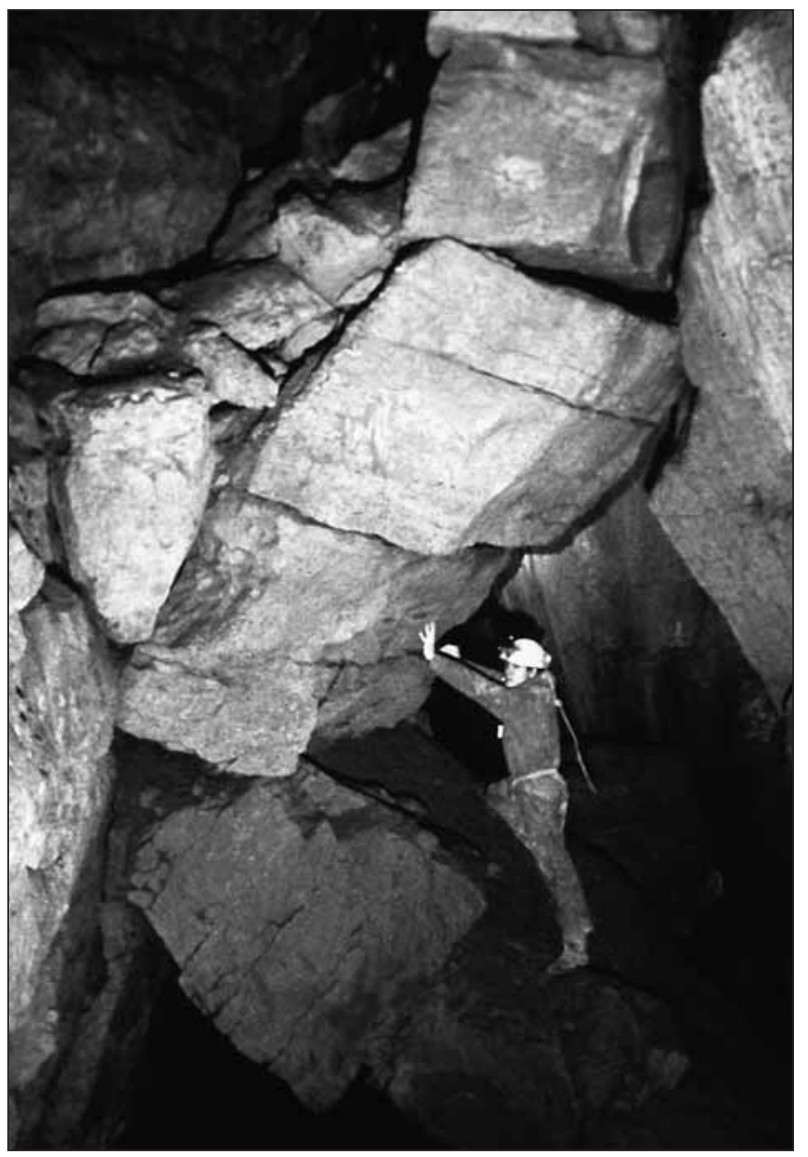

Photo 6 :

Event de la Vernière : "le pilier de l'Apocalypse". Le recreusement du remplissage provoque une détente des parois, premier stade vers la formation de galeries à section carrée (cliché S. Bruxelles). "Pilar of Apocalypse" in the "Event de la Vernière". The erosion of cave filling provokes the walls distension and the formation of galleries with "square section". 
On peut situer la mise en place de ce nouveau karst en fonction d'un potentiel généré par la surrection générale et le creusement des vallées régionales, c'est-à-dire à partir du Miocène supérieur [Ambert et Ambert, 1995].

\section{Fonctionnement actuel}

Certaines cavités perchées, situées à faible profondeur sous les crêtes calcaires, sont altitudinalement très loin du niveau des circulations, parfois à plus de 400 m. Les infiltrations qui traversent ces cavités fossiles percolent à travers le remplissage. Les colorations ont montré que certains de ces lambeaux de cavités perchées, comme l'aven du Calabrien et la grotte de Valaurie, sont drainés par des circulations actives proches du niveau de base. Leur drainage s'opère le plus souvent par le biais de diaclases plus récentes, probablement dues à la détente du massif et le long de joints de strates (grotte de Rouville). Elles sont ensuite collectées soit dans des galeries actives plus jeunes et de morphologie très différente (rivière de Trabuc), soit dans une de ces anciennes galeries en cours de décolmatage (évent de la Vernière). Enfin, quelques cavités recoupées par le Gardon ont par ailleurs fonctionné en perte de cette rivière. Les traces de cette activité viennent s'ajouter à celles des phases précédentes. De belles coupes de sédiments, comprenant une proportion importante de sables micacés, témoignent de cette activité parfois plus de $100 \mathrm{~m}$ au-dessus du niveau actuel du Gardon (grotte de Trabuc, grotte de Valaurie). Ce phénomène est toujours fonctionnel comme nous avons pu le vérifier dans les niveaux bas de Trabuc durant l'été 94 [Bruxelles, 1994].

\section{CONCLUSION}

Le karst du bassin de Mialet présente donc plusieurs éléments d'âges différents. Il comprend des fragments d'anciens réseaux, véritables paléokarsts, probablement formés sous couverture avant la subsidence oligocène, et des cavités plus jeunes aux bassins d'alimentation plus réduits qui recoupent et réutilisent en partie ces vieilles cavités. Leur formation est directement liée à la mise en place d'un nouveau réseau hydrographique et au démantèlement du massif dès le Miocène supérieur. L'étude approfondie des remplissages karstiques devrait nous permettre de préciser l'évolution de ce karst. Par extension, nous espérons obtenir des informations sur la paléogéographie et donc le mode de fonctionnement de ces cavités. La découverte récente sur le sommet des reliefs, de lambeaux de formations superficielles (argile à chailles) comparables à celles que nous étudions en Aveyron sur le plateau du Larzac devrait aussi apporter des précisions supplémentaires concernant ces questions [Bruxelles, 1995]. Lorsque, par érosion régressive, le Gardon d'Anduze aura franchi le seuil granitique du Roucan, le niveau de base s'abaissera encore dans le bassin de Mialet. Les galeries actives seront en partie abandonnées par les eaux au profit d'autres plus profondes. En parallèle, l'enfoncement des ruisseaux affluents de surface fractionnera encore plus le massif calcaire. Les éléments du karst désormais isolés les uns des autres évolueront indépendamment et devront de nouveau se réorganiser à une échelle encore plus locale.

Remerciements : Je remercie tout particulièrement Jean Nicod, Martine et Paul Ambert, Jean-Louis Guendon, Yves Quinif et Jacques Schroeder pour leur soutien et leurs précieux conseils. J'exprime également ma gratitude à Stéphane Bruxelles, Aimé Mallet, Henri Paloc et Michel Wienin.

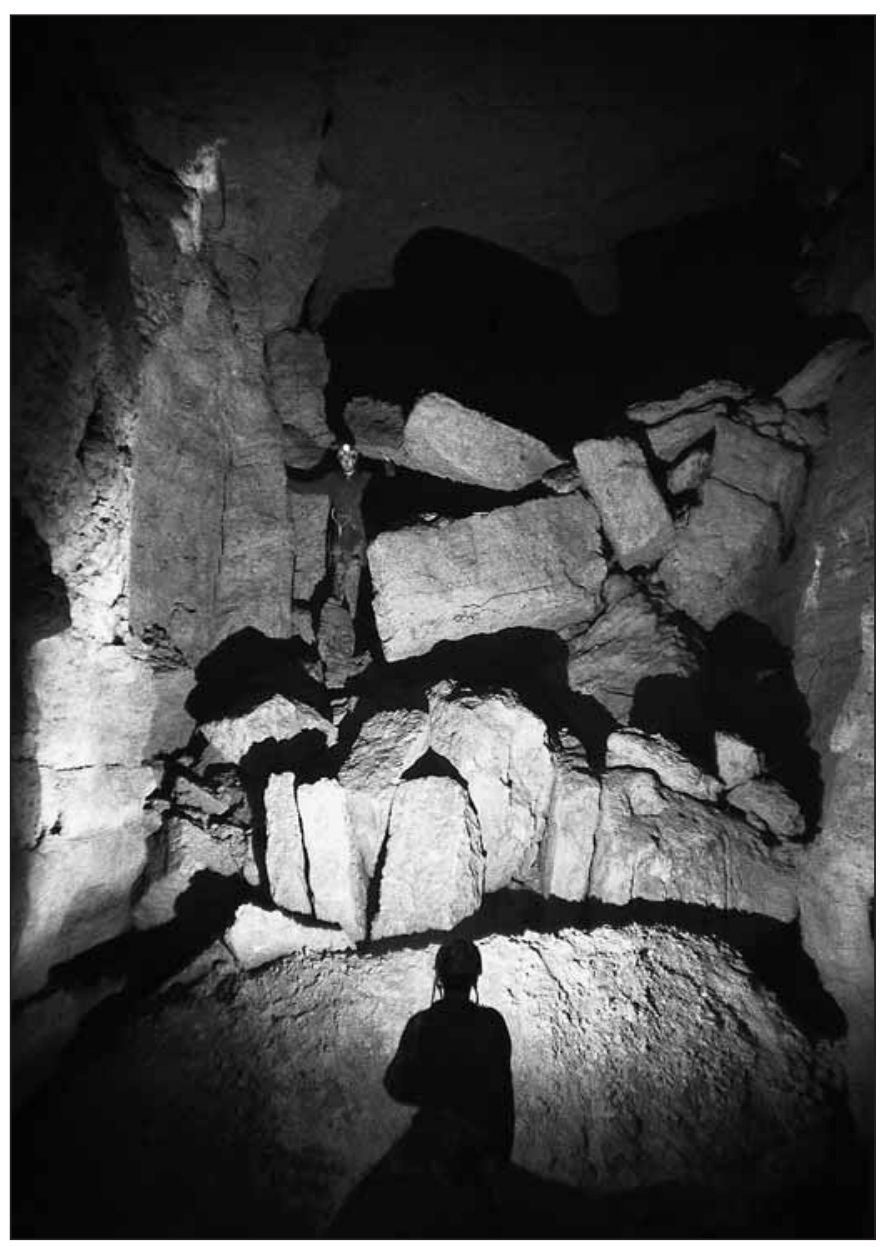

Photo 7 : Galerie de la Trémie dans l'Event de la Vernière.

Le sol est constitué de blocs effondrés sur $15 \mathrm{~m}$ d'épaisseur. La galerie a déjà acquis une "section carrée" (cliché L.B.). "Galerie de la Trémie" in the "Event de la Vernière". The floor is constituted of 15 meters height of collapsed blocks. It's now a "square section" gallery.

\section{BIBUOGRAPHIE}

\section{AMBERT P. et AMBERT M., 1995}

Karstification des plateaux et encaissement des vallées au cours du Néogène et du Quaternaire dans les Grands Causses méridionaux (Larzac, Blandas). Géologie de la France, nº ${ }^{\circ}$, p. 37-50.

AMBERT P., COULET E., FABRE G., GUENDON J.L., NICOD J., ORENGO C., 1978 - Le causse de Blandas et les gorges de la Vis : Etude géomorphologique. Coédition C.E.R.G.A.-E.R.A 282., Grenoble, 52 p. (C.E.R.G.H. Mémoire n 13 , Travaux E.R.A. 282, ${ }^{\circ} 7$ )

BRUXELLES L., 1994 - Géomorphologie des karsts du bassin Mialet (Gard) : Données actuelles. Université Paul Valéry, Montpellier, 128 p (Mémoire de maîtrise)

BRUXELLES L., 1995 - Etude des altérites, des formations superficielles et endokarstiques $d u$ causse de l'Hospitalet, secteur oriental (Aveyron) : Conséquences géomorphologiques et contraintes pour l'aménagement. Université de Provence, Montpellier, 70 p. (Mémoire de DEA)

COLAS R et RUHLAND, M., 1982 - Tectonic control of the karstic network, in the Trabuc cave. In : Actes du deuxième Symposium International sur l'Utilisation de l'Espace Karstique, Bari, 20-22 Mai 1982, p. 133-146.

PERRISSOL M., 1990 - Sédimentologie et métallogénie du Lias carbonaté de la bordure cévenole. Université des Sciences et Technologies Littéraires, Montpellier, 606 p. (Thèse)

RENAULT P., 1993 - Géologie du bathyphréatisme, morphométrie, soutirages dans le nord de la France et dans le Quercy. In : Actes des rencontres d'Octobre, $n^{\circ} 3$, p. $91-96$.

Société Cévenole de Spéléologie et de Préhistoire, 1988.- Monographie de la grotte de Trabuc. 96 p. 